\title{
Evaluation of Fracture Characteristics of Random Field with Anisotropic Spatial Correlation
}

\author{
Noriyuki Kobayashi $^{1}$, Yoshitaka Yoshitake ${ }^{2}$ and Kenji Iima ${ }^{3}$
}

\begin{abstract}
Soil property varies randomly in a field and there is some correlation between its values at any two points. Especially, in an artificial soil structure like an embankment, it has some anisotropic spatial correlation due to constructive features. In our earlier work, the effect of the standard deviation and the correlation distance of soil permeability on total flow rate in a random field with isotropic spatial correlation has been clarified and spatial spanning of lower or higher permeability that forms "impervious wall" or "flow path" has been reproduced by Percolation theory. This paper attempts to evaluate objectively fracture characteristics of random field with anisotropic spatial correlation by using fractal dimensions. Spatially correlated random fields with anisotropic spatial correlation distance are generated, and fractal dimensions are examined for each of these random fields. As a result, the fractal dimensions are shown to have some close relation to the anisotropic spatial correlation in random fields.
\end{abstract}

Keywords: Fractal dimension; Random field; Anisotropic spatial correlation

\section{Introduction}

Soils exhibit highly variable properties in a field. When they are spatially distributed, there is some correlation between their values at any two points. The values at any two points are almost the same if the points of interest are closely located. On the contrary, correlation is reduced as the distance between the points increases. To describe the spatial correlation of hydraulic property, some geostatistical covariance models have been proposed over the past few decades (e.g., Fenton and Vanmarcke (1990) for exponential model, Bellin and Rubin (1996) for power model, Saito and Kawatani (2000) for $1 / f$ model.).

The authors (2001) generated soil permeability distributions employing the covariance function of exponential type with isotropic spatial correlation, and investigated flow properties that result from a statistical approach to confined seepage problem. As the standard deviation of permeability coefficient $\sigma_{k}$ increases, the mean flow rate $Q_{m}$ decreases and the variance of flow rate $\sigma_{f}^{2}$ increases. This is due to the fact that the portion of lower or higher permeability becomes dominant, that is, lower or higher permeability cells concentrate in a particular part and are arranged like an impervious wall or a flow path, respectively.

The authors (2002) also evaluated the "impervious wall" and the "flow path" by using percolation theory. Random permeability fields with isotropic spatial correlation distance $a$ were generated, and finite element seepage analysis was performed for each of these random fields to examine percolation properties. And, the relationship between the total flow rate analyzed by FEM and the indexes of percolation,

\footnotetext{
${ }^{1}$ Associate Professor, Faculty of Agriculture, Ehime University, 3-5-7 Tarumi, Matsuyama, Ehime, 790-8566 Japan

${ }^{2}$ Professor, Faculty of Agriculture, Ehime University, 3-5-7 Tarumi, Matsuyama, Ehime, 790-8566 Japan

${ }^{3}$ Engineer, Kagawa Prefecture Government, 4-1-10 Bancho, Takamatsu, Kagawa, 7760-8570 Japan
}

namely, threshold, threshold probability and cluster size, was derived, and the threshold and threshold probability were shown to have a significant effect on flow rates.

On the contrary, it is well known that soil properties in an artificial soil structure like an embankment have some anisotropic spatial correlation due to constructive features. In this paper, we intend to evaluate objectively fracture characteristics of random field with anisotropic spatial correlation by using fractal dimensions (Mandelbrot, 1967). First, 100 sets of random fields with mean $\mu_{k}$, standard deviation $\sigma_{k}$ and anisotropic spatial correlation distance $a$ are generated, and fractal dimensions are calculated for each of these random fields. And, the relationship between the anisotropic spatial correlation distance $a$ and fractal dimensions $\left(F D, A F D_{x}\right.$ and $\left.A F D_{y}\right)$ is discussed. As a result, the fractal dimensions can represent characteristics of the covariance function with anisotropic spatial correlation distance.

\section{Fractals and fractal dimensions}

\subsection{Fractals}

Fractals provide a mathematical rule for dealing with irregular and complex shapes that display similar patterns or characteristics over a range of scales (Rodriguez-Iturbe and Rinaldo, 1997). The concept of fractals was introduced by Mandelbrot (Mandelbrot, 1967) and characterizes the objects in which properly scaled portions are identical to the original object.

Self-similarity is a concept associated with fractal geometry that refers to invariance with respect to multiplicative changes. An object is said to be selfsimilar if it looks "almost" the same on any scale, and a self-similar object can be divided more small structures under closer scrutiny. The small structures are identical to the larger scale ones when they undergo 
an expansion in their scale. The self-similarity is described by a property called scaling or dimension.

\subsection{Fractal dimensions}

The length of a complex curve can be measured with stepping along it with a ruler length $r$, if one uses the following relationship:

$$
L=C \cdot r^{1-F D}
$$

where $C$ : a constant, $r$ : the ruler length, and $F D$ : a constant.

A relationship between the number of steps $N$, the ruler length $r$ and the curve length $L$ can be obtained from:

$$
L=N r
$$

The following equation can be written with eliminating $L$ from (1) and (2):

$$
N=C \cdot r^{-F D}
$$

(3) indicates that a plot of the quantity on a log-log graph of length $N$ versus inverse number of ruler size $1 / r$ gives a straight line. The slope $F D$ is the most common definition of fractal dimension:

$$
F D=\lim _{r \rightarrow 0} \frac{\log N(r)}{\log (1 / r)}
$$

The fractal dimension $F D$ is sometimes assumed equal to the so-called capacity dimension.

\subsection{Box counting method}

The box counting dimension is applied to the natural sciences most frequently. The dimension is calculated easily even in the case of objects in higher dimensional spaces (e.g., in a three dimensional case the boxes are cubes with side size $r$ ). And, it can be assumed that for all practical purposes capacity and box counting dimensions coincide in the applications pursued here.

An image is covered by some boxes of side size $r$, and the number of boxes needed to cover the image is determined. A relationship between the number of boxes $N$, the box of side size $r$ and the box counting dimension can be obtained from:

$$
N(r)=\frac{1}{r^{F D}}
$$

To estimate the box counting dimension, the Euclidean space containing the image can be divided into a grid of boxes of side size $r$ and the image can be divided into the counting boxes $N(r)$ which are not empty. Then $r$ is changed to progressively smaller sizes and the corresponding number of non-empty boxes $N(r)$ is counted (Figure 1). A relationship of the logarithm of $N(r)$ versus the logarithm $1 / r$ gives a straight line whose slope corresponds to the box counting dimension (Figure 2).

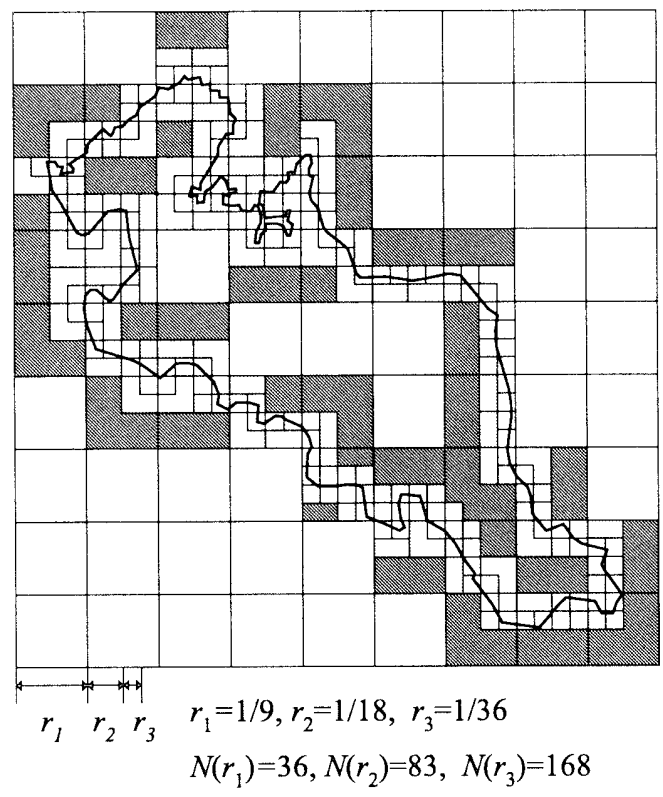

Figure 1: Boxes needed to cover an image

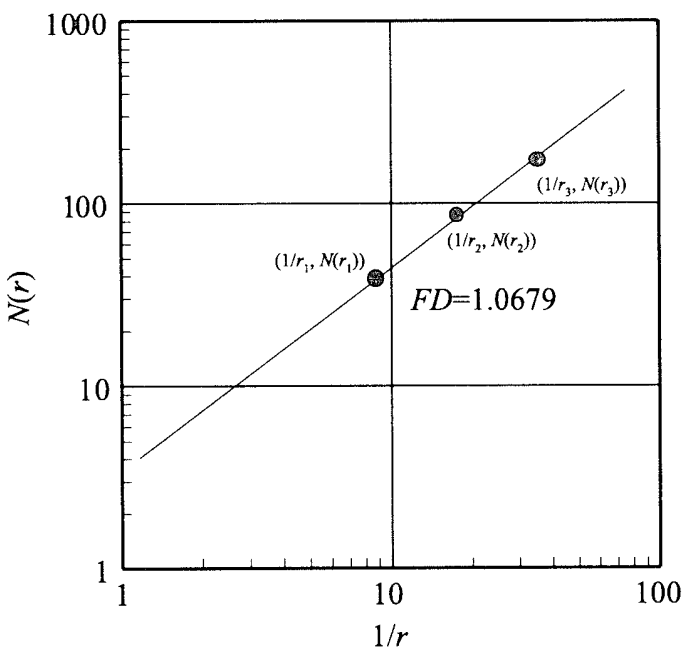

Figure 2: Number of boxes $N(r)$ versus its side size $r$ for the image in Figure 1

\section{Process for generation of Gaussian random} numbers

A structure of spatial distribution for soil permeability can be represented by a covariance function that describes the variability between two points. The covariance function employed herein is an exponential type in two dimensions expressed in the following form (Journel (1978), Hamaguchi (1998)):

$$
C\left(h_{x}, h_{y}\right)=\sigma_{g}^{2} \exp \left\{-\sqrt{\left(\frac{h_{x}}{a_{x}}\right)^{2}+\left(\frac{h_{y}}{a_{y}}\right)^{2}}\right\}
$$

where $C(\cdot)$ : covariance function, $\sigma_{g}$ : global standard deviation, $h_{x}, h_{y}$ : separated distances between a pair of any two points in the $x$ and $y$ directions, respectively, $a_{x}, a_{y}$ : correlation distances in the $x$ and $y$ di- 
rections, respectively, and the subscripts $x, y$ : Cartesian coordinates. A random permeability field with anisotropic spatial correlation can be generated when the different correlation distances $a$ are given in the $x$ and $y$ directions.

A Gaussian random number $Z$ is generated with the covariance function (6) and is defined as the average of $G(x, y)$ over the intervals $s \leq x \leq t$ and $u \leq y \leq v$ :

$$
Z=\frac{1}{(t-s)(v-u)} \int_{s}^{t} \int_{u}^{v} G(x, y) d x d y
$$

A whole domain where $Z$ is generated is determined. First, a global mean $\mu_{g}$ for the domain is defined. Next, the domain is subdivided into 4 equal cells subject to the rule that their values must average $\mu_{g}$ and be correlated each other according to (6). At the subsequent step, each parent cell generated at the previous step is subdivided into 4 equal cells due to the same rule that their values average the parent mean (Fenton and Vanmarcke (1990)).

A random field with spatial correlation structure can be generated using the method of postmultiplying the Gaussian random number vector by the upper triangular Cholesky decomposition of the covariance matrix obtained with covariance function (6) (Kobayashi, 2001).

\section{Estimation of fracture characteristics}

\subsection{Calculation of fractal dimension}

The Gaussian random numbers obtained by the above mentioned method are defined at the centroid of each cell, and the fractal dimensions of the following curved surface are calculated.

$$
Z_{l, m}=f\left(x_{l, m}, y_{l, m}\right) \quad l, m=1,2, \cdots, n
$$

where $Z_{l, m}$ : Gaussian random number of the $l, m$ th cell, $n$ : the number of row or column, $x_{l, m}, y_{l, m}$ : the coordination of the centroid of the $l, m$-th cell. Figure 3 illustrates a sample curved surface with $\mu_{g}=0.0, \sigma_{g}=1.0, a_{x}=a_{y}=0.4$, and $n=64$. In this case, the boxes for box counting are cubes with side size $r\left(=2^{-\imath}\right)$. The integer number $i$ is in the wide range of 1 to 8 . This fractal dimension $F D$ lies between 2 and 3, and approximates 2 as the curved surface is flatter.

Two new fractal dimensions are introduced, as $F D$ cannot distinguish the anisotropy of the Gaussian random numbers surface. The fractal dimensions $A F D_{x}$ and $A F D_{y}$ as shown in Figure 4 are applied to clarify the anisotropy, and are defined as:

$$
A F D_{x}=\frac{1}{n} \sum_{\imath=1}^{n} F D_{x \imath} \quad A F D_{y}=\frac{1}{n} \sum_{i=1}^{n} F D_{y \imath}
$$

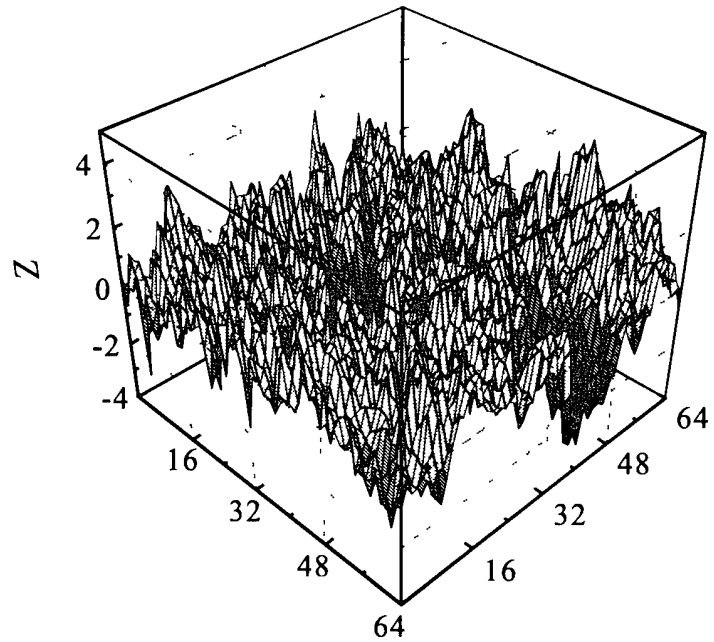

Figure 3: Sample curved surface of Gaussian random numbers $Z$

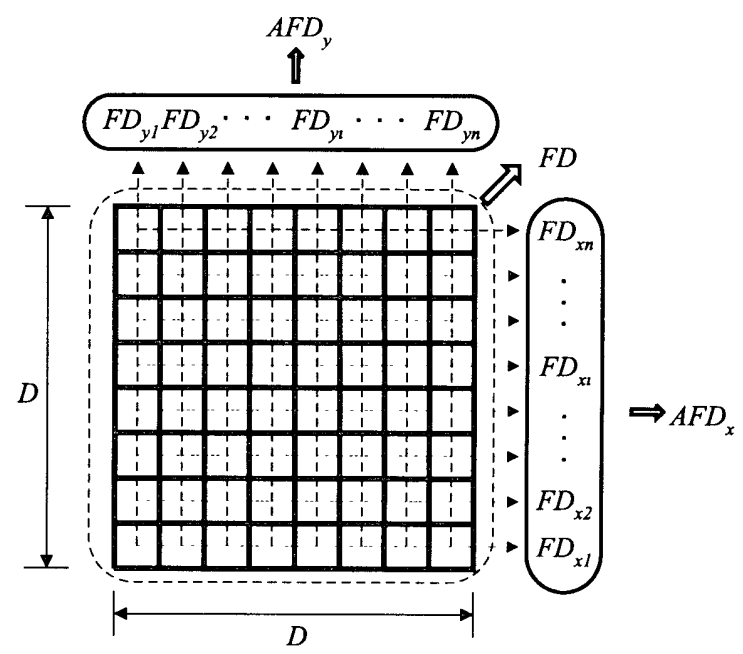

Figure 4: Schematic diagram of $A F D$

where $i$ : row or column number and $F D_{x i}, F D_{y i}$ : the fractal dimensions of the following curves, respectively.

$$
\begin{array}{r}
k_{\imath, m}=f\left(x_{\imath, m}, y_{\imath, m}\right) \quad m=1,2, \cdots, n \\
k_{l, \imath}=f\left(x_{l, \imath}, y_{l, \imath}\right) \quad l=1,2, \cdots, n
\end{array}
$$

This fractal dimensions $A F D_{x}$ and $A F D_{y}$ lie between 1 and 2 , and closely estimated at 2 as the curve is ruggeder.

\subsection{Simulation procedure}

A relationship between the fracture characteristics and the anisotropic spatial correlation in a random field is investigated, taking the following procedure.

1. A global mean $\mu_{g}$, a global standard deviation $\sigma_{g}$ and correlation distances $a_{x}$ and $a_{y}$ are given and a set of random numbers $Z$ in a whole domain is generated. 


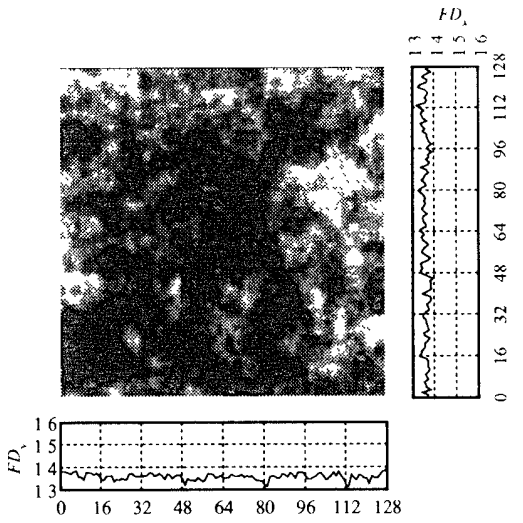

(a) $a_{x}=0.25, a_{y}=0.25$

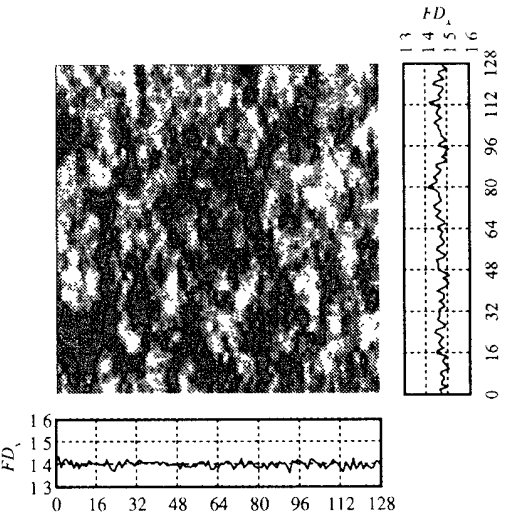

(b) $a_{x}=0.05, a_{y}=0.25$

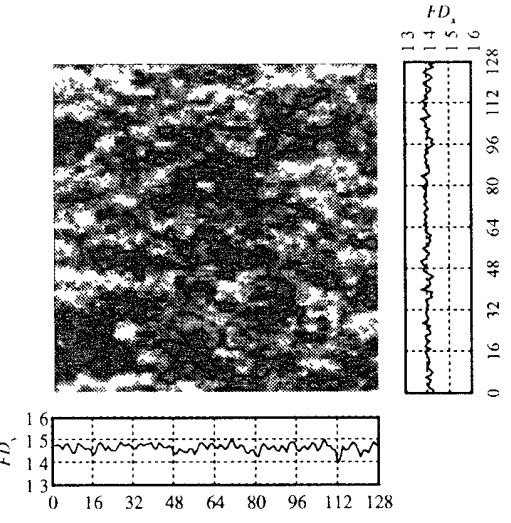

(c) $a_{x}=0.25, a_{y}=0.05$

Figure 5: Spacial distributions of $Z$

Table 1: Calculated fractal dimensions

\begin{tabular}{c|cc|ccc}
\hline Case & $a_{x}$ & $a_{y}$ & $F D$ & $A F D_{x}$ & $A F D_{y}$ \\
\hline \hline $\mathrm{A}$ & 0.25 & 0.25 & 2.43 & 1.36 & 1.36 \\
$\mathrm{~B}$ & 0.05 & 0.25 & 2.52 & 1.47 & 1.40 \\
$\mathrm{C}$ & 0.25 & 0.05 & 2.52 & 1.40 & 1.47 \\
\hline
\end{tabular}

2. Fractal dimensions $F D, A F D_{x}$ and $A F D_{y}$ of the random field generated at step 1 are obtained.

3. A relation between the fracture characteristics and the anisotropic correlation distance $a$ is derived.

$Z$ is the random number with $\mu_{g}=0.0, \sigma_{g}=1.0$, and the length of the model $D$ in Figure 4 is 1.0. The parametric studies are designed to show the effect of anisotoropic correlation distances $a_{x}$ and $a_{y}$. As the values of $a_{x}$ and $a_{y}, 0.01,0.025,0.05,0.1,0.25,0.5$, $1.0,2.5,5.0$, and 10.0 are considered. The domain is divided into $16,384(128 \times 128)$ cells.

\subsection{Results and discussion}

Typical spatial distributions with the following correlation distances $a\left(a_{x}, a_{y}\right)$, namely, Case $\mathrm{A}\left(a_{x}=\right.$ $\left.0.25, a_{y}=0.25\right)$, Case B $\left(a_{x}=0.05, a_{y}=0.25\right)$, and Case C $\left(a_{x}=0.25, a_{y}=0.05\right)$, selected from 100 cases, are shown in Figure 5 in which the fractal dimensions $F D_{x}$ and $F D_{y}$ are written together. The cells are gradated from black to white as the Gaussian random number $Z$ is increased. $F D, A F D_{x}$ and $A F D_{y}$ in each case are summarized in Table 1. As is clear from Figure 5, the random numbers are strongly correlated in the field and the cells with almost the same value concentrate in a particular part in all cases. In Case $\mathrm{A}$, the concentrations are arranged in isotoropic condition and $A F D_{x}$ is equal to $A F D_{y}$. In Cases $\mathrm{B}$ and $\mathrm{C}$, the arrangement of the concentrations is anisotropic and $A F D_{x}$ and $A F D_{y}$

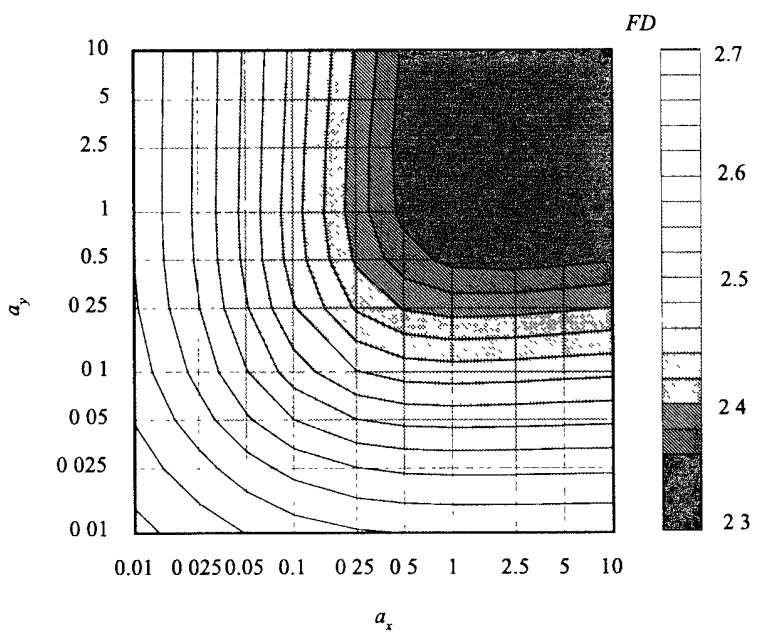

Figure 6: Relation between $F D$ and $a$

are different. As $a$ in a direction decreases, the length of the concentrations in that direction is smaller and $A F D$ in the direction is larger than $A F D$ in the other direction.

Figure 6 shows a relationship between $F D$ and $a$. $F D$ becomes larger as $a$ is smaller. And, $F D$ tends to decrease, as the fluctuation of $Z$ in the cells closely located decreases, namely, as $a$ increases. The tendency remarkably appears in the case of isotropic correlation distance $a\left(a_{x}=a_{y}\right)$.

Figure 7 shows a relationship between $F D$ and $a_{x}$ in each $a_{y}$. When $a_{y}$ is fixed, $F D$ decreases with the increasing $a_{x}$. This reduction rate becomes larger as $a_{y}$ increases and $F D$ s are almost the same with the increasing $a_{x}$ when $a_{y}$ is larger than $D$. And, $F D$ is fairly constant when $a_{x}$ is larger than $D$. If $a_{x}$ is fixed in turn and $a_{y}$ changes, the results are indistinguishably different. The relationship between $F D$ and $a$ demonstrates characteristics of the covariance function (6).

A relationship between $A F D_{x}$ and $a$ are shown 


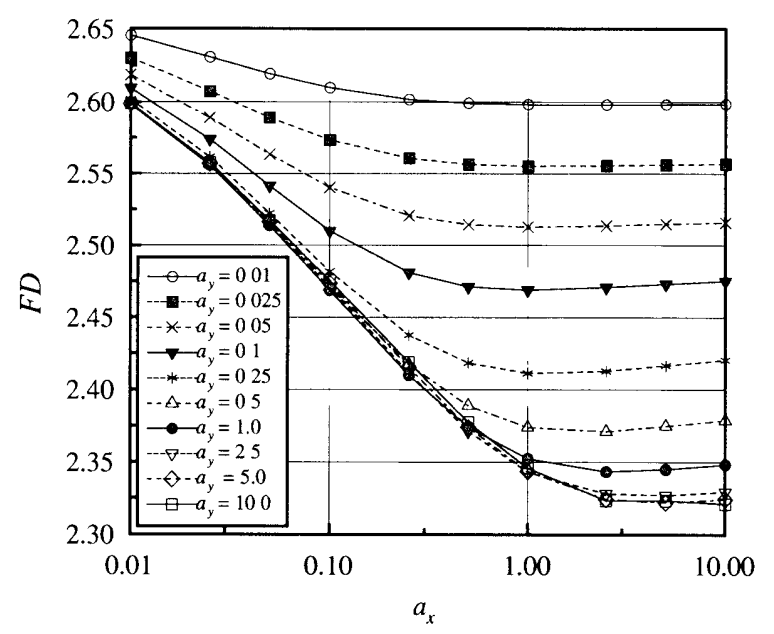

Figure 7: Relation between $F D$ and $a_{x}$ in each $a_{y}$

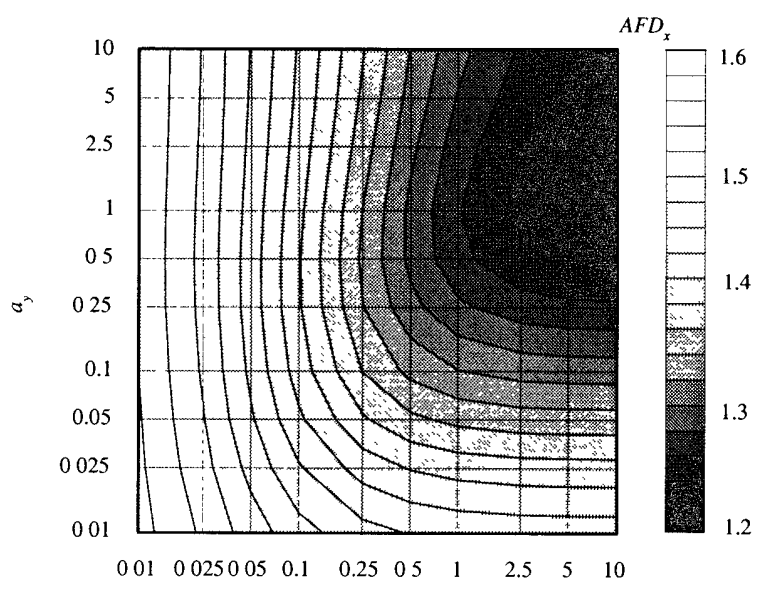

$a_{x}$

Figure 8: Relation between $A F D_{x}$ and $a$

in Figure 8. $A F D_{x}$ distribution lacks the symmetry that $F D$ distribution displays in Figure 6. Figure 9 shows effects of $a_{x}$ and $a_{y}$ on $A F D_{x}$. Figure 9(a) indicates that $A F D_{x}$ decreases with the increasing $a_{x}$. This reduction rate becomes larger than the rate of $F D$ in Figure 7. And, when $a_{x}$ is larger than $D$ and $a_{y}$ is less than $0.25, A F D_{x}$ is constant. But, when $a_{y}$ is larger than $0.5, A F D_{x}$ keeps decreasing with the increasing $a_{x}$ even if $a_{x}$ is larger than $D$. Figure 9 (b) demonstrates that $A F D_{x}$ decreases with the increasing $a_{y}$, but the reduction rate is smaller than that in Figure 9(a). Especially, when $a_{x}$ is less than $0.05, A F D_{x}$ is fairly constant. And, $A F D_{x}$ slightly increases with the increasing $a_{y}$ when $a_{y}$ is larger than $D$. For the reasons mentioned above, $A F D_{x}$ depends on $a_{x}$ more heavily than on $a_{y}$. If the vertical axis $A F D_{x}$ is exchanged for $A F D_{y}$, the results have almost the reverse tendency in terms of $a_{x}$ and $a_{y}$. The relationship between $A F D$ and $a$

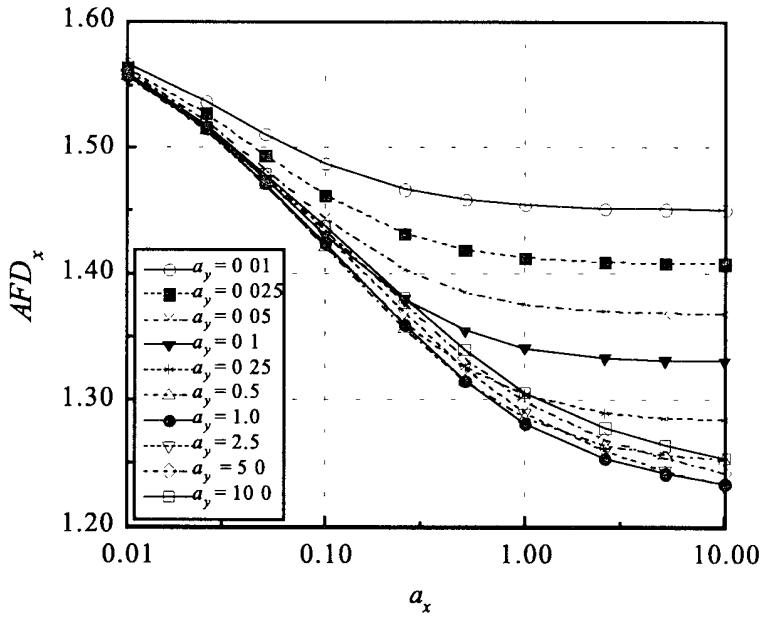

(a) Relation between $A F D_{x}$ and $a_{x}$

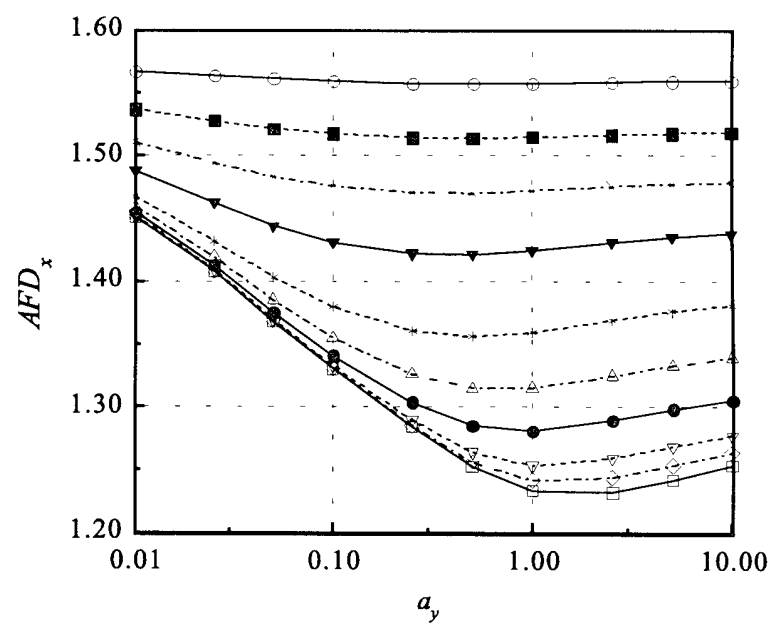

(b) Relation between $A F D_{x}$ and $a_{y}$

Figure 9: Effect of $a$ on $A F D_{x}$

also shows characteristics of the covariance function (6), and a set of $A F D_{x}$ and $A F D_{y}$ can represent anisotropic spatial correlation.

\section{Conclusions}

With generation of heterogeneous random fields having anisotropic spatial correlation, we have attempted to estimate the fracture characteristics. Fractal analyses with random fields of 100 cases have been performed to investigate the relationship between correlation distance $a$ and fractal dimensions $F D, A F D_{a}$ and $A F D_{y}$. The conclusions drawn from this study are as follows:

1. As $a$ increases, $F D$ and $A F D$ decreases, and the reduction rate decreases with the increasing $a$.

2. $F D$ and $A F D$ are fairly constant or the rate of change is smaller with the increasing $a$ when $a$ is larger than the length of the model $D$. 
3. $A F D_{x}$ and $A F D_{y}$ depend heavily on $a_{x}$ and $a_{y}$, respectively.

4. The relationship among $F D, A F D$ and $a$ also shows characteristics of the covariance function, and a set of $A F D_{x}$ and $A F D_{y}$ can represent anisotropic spatial correlation.

\section{References}

[1] Bellin, A. and Rubin, Y. (1996): HYDRO_GEN: A new random number generator for correlated properties, Stochastıc Hydrology and Hydraulıcs, Vol. 10, No. 4, pp. 253-278.

[2] Fenton, G. A. and Vanmarcke, E. H. (1990): Simulation of random fields via local average subdivision, J. Engineering Mechanics, ASCE, 116(8), pp.17331749.

[3] Hamaguchi, T. (1998): Studies on Inverse Problems Relating to Design for Underground Dam through New Modeling for Groundwater Flow with Moving Boundaries, Doctoral dissertation, Kyoto University, pp.91-98.
[4] Journel, A G. and Huijbregts, C J. (1978): Mınıng Geostatıstıcs, Academic, San Diego, Calif., pp.161195.

[5] Kobayashi, N., Yoshitake, Y. and Iima, K. (2001): A Statistical Approach to Confined Seepage Problem with Generation of Random Permeability Field, $J$. Rainwater Catchment Systems, 7(1), pp.7-12.

[6] Kobayashi, N., Yoshitake, Y. Otsuka, N., Iima, K and Matsumoto, S. (2002): Percolation Properties of Random Permeability Field, J. Rainwater Catchment Systems, 7(2), pp.7-11.

[7] Mandelbrot, B. B. (1967): How long is the coast of Britain? Statistical self-similarity and fractional dimension, Scrence, No. 156, pp.636-638.

[8] Rodriguez-Iturbe, I and Rinaldo, A. (1997): Fractal river basins - Chance and self-organization - , Cambridge unvversity press, pp.99-106.

[9] Saito, M. and Kawatani, T. (2001): Study on applicability of geostatistical models of hydraulic conductivity, J. Geotechnical Engineering, JSCE, No. 696(III-57), pp.245-258. (in Japanese).

[Discussion open until December 31, 2003] 